\title{
Preditores da empatia dirigida a humanos e outros animais em portugueses e anglo-saxónicos
}

\author{
Ana Emauz ${ }^{1}$, Augusta Gaspar ${ }^{2} \&$ Francisco Esteves ${ }^{3}$ \\ ${ }^{1}$ Centro de Investigação e Intervenção Social (CIS-IUL), Escola de Ciências Sociais e Humanas do ISCTE-IUL \\ ${ }^{2}$ Católica Research Centre for Psychological, Family and Social Wellbeing (CRC-W), Faculdade de Ciências \\ Humanas, Universidade Católica Portuguesa/Centro de Investigação e Intervenção Social (CIS-IUL), ISCTE- \\ IUL \\ ${ }^{3}$ Department of Psychology, Mid Sweden University, Östersund, Suécia/Centro de Investigação e Intervenção \\ social (CIS-IUL), ISCTE-IUL, Lisboa
}

\begin{abstract}
Resumo: A relação entre empatia dirigida a humanos e empatia dirigida a outros animais tem sido reportada como fraca, sendo pouco conhecidos os fatores que predizem a segunda. Neste estudo, examinaram-se potenciais variáveis preditoras de cada uma destas duas formas de empatia e compararam-se participantes lusófonos e anglo-saxónicos, inspecionando possíveis especificidades culturais. Foi conduzido um inquérito na web que incluiu as versões portuguesa e inglesa de uma escala de empatia para com humanos e de uma escala de empatia para com animais, bem como questões relacionadas com animais de estimação, religião, dieta e participação em ONGs. A testagem de modelos de regressão múltipla evidenciou o género como preditor da empatia com humanos, mas apenas no grupo lusófono. 0 sexo feminino e a vivência com animais de estimação foram preditores de empatia para com animais em ambos os grupos; a ligação a ONGs também o foi na população lusófona, enquanto que na anglo-saxónica pesou mais a dieta vegetariana/vegana.
\end{abstract}

Palavras-chave: Preditores de empatia; empatia para com animais; empatia para com humanos; animais de estimação; escala de empatia para com animais; escala de empatia para com humanos.

Predictors of empathy towards humans and animals in a Portuguese and English speaking population: While the relation between human directed empathy (HDE) and animal directed empathy (ADE) has been reported as relatively weak, very little is known on factors underpinning the development of the later. We explored predictive models of HDE and ADE regressing them on gender, religion, diet, having pets and belonging to NGO charities, and we also compared portuguese with english speaking people as a proxy of culturally distinct populations. An online survey was conducted and included portuguese and english versions of measures of HDE and ADE, as well as questions targeted at the predictive variables. Results indicated gender as the single predictor of human directed empathy in the "Portuguese" group whereas both gender and having pets predicted empathy towards animals in both groups. NGO affiliation is a predictor of empathy towards animals in the portuguese but not in the english group, where a vegetarian/vegan diet is the strongest predictor.

Keywords: Empathy predictors; empathy towards animals; empathy towards humans; IRI; AES.

A empatia é um tema crescente na literatura científica, vindo gradualmente a ganhar terreno na comunicação social, pela visibilidade da sua importância nas relações sociais, principalmente enquanto mecanismo motivador de confiança e de comportamentos pro-sociais como o altruísmo (de Waal, 2008). A visão dominante, embora não a única, é a que desempenha um papel crucial na coesão e sobrevivência de grupos onde os indivíduos se conhecem entre si (Castro, Gaspar, \& Vicente, 2010; Gaspar, 2014; Preston \& de Waal, 2002).

A empatia é um construto multidimensional que inclui uma componente cognitiva e outra emocional. A empatia cognitiva está relacionada com a capacidade de nos colocarmos no lugar do outro, de entender as suas emoções e experiências, enquanto que a empatia emocional implica a partilha do estado emocional de uma outra pessoa (Smith, 2006). Se, por um lado, a empatia nos permite reagir de forma mais adequada às diversas situações e gerir as relações interpessoais (Eisenberg et al., 1989; Eisenberg, Miller, Shell, McNalley, \& Shae, 1991), por outro, a falta de empatia também está relacionada com comportamentos agressivos, como a violência doméstica, o bullying ou com a psicopatia (American

${ }^{1}$ Morada para correspondência: Centro de Investigação e Intervenção social (CIS-IUL), Escola de Ciências Sociais e Humanas do ISCTE-IUL, Av. das Forças Armadas, 1649-026 Lisboa, Portugal. E-mail: aemauz@gmail.com 
Psychiatric Association, 2013; Ascione, Weber, \& Wood, 1997; McPhedran, 2009; Thompson \& Gullone, 2008).

Parecendo claros os benefícios da empatia dirigida a conspecíficos, a orientação da empatia para seres de outras espécies é menos óbvia. E, apesar de reconhecida a estreita ligação que se pode desenvolver com os animais de estimação, ainda se sabe pouco sobre os fatores que subjazem à capacidade de sentirmos empatia por membros de outra espécie, e sobre como esta se relaciona com a empatia que sentimos pelos humanos. Surge neste contexto o presente trabalho, que tem como principal objetivo examinar alguns possíveis preditores da empatia e averiguar se os fatores que predizem empatia dirigida a humanos são os mesmos que predizem a empatia dirigida a outros animais. Esta é uma questão em aberto e para a qual existe um grande hiato na literatura. Pretende-se também aqui apurar se estes fatores são transversais a pessoas de diferentes origens geográficas e culturais, comparando pessoas que falam a língua portuguesa com pessoas anglo-saxónicas (assumindo que os respondentes da mesma língua partilham aspetos culturais comuns e que as diferentes línguas expressam também diversidade cultural).

\section{Empatia dirigida a humanos e a outros animais}

A forma como nos sentimos afetados emocionalmente está associada não só à intensidade do estímulo que percecionamos, mas também ao alvo em questão, à sua relevância emocional, havendo evidência que sugere que os seres humanos experimentam uma maior empatia pelas espécies filogeneticamente mais próximas (Westbury \& Neumann, 2008). Há presentemente estudos que mostram, por um lado, que os humanos são capazes de sentir empatia pelos animais (e.g., Emauz, Gaspar, Esteves, \& Carvalhosa, 2016; Paul, 2000), e por outro, que a preocupação com o sofrimento/bem-estar dos animais está associada a uma maior empatia para com humanos (Ascione, 1992; Ascione \& Webber, 1996; Komorosky \& 0`Neal, 2015). 0 facto de os humanos poderem sentir empatia pelos animais, sobretudo pelos animais de estimação, poderá ajudar a explicar os laços fortes que se formam com os últimos.

Para de Waal (2008), sentir empatia para com os outros animais parte da generalização de um mecanismo que evoluiu no contexto dos cuidados maternos e da resposta a sinais do bebé/cria, e que se alargou a outros conspecíficos e até a animais de outras espécies. Assim, a empatia por outros animais terá sido desencadeada por processos emocionais semelhantes aos utilizados na interação com humanos. A empatia é inicialmente (quer do ponto de vista filogenético quer ontogenético) um fenómeno de natureza emocional, o que é amplamente corroborado por dados do desenvolvimento neurológico (e.g. Decety \& Svetllova, 2012; Schore, 2001). Outros investigadores dão à componente cognitiva da empatia um papel mais importante no desenvolvimento da empatia humana. Por exemplo, para Hydr (2011), a empatia humana nasce sobretudo da alomaternidade (a distribuição dos cuidados parentais por outros que não a mãe), algo raro em primatas não-humanos mas comum na nossa espécie, a que subjaz a capacidade de confiar e de avaliar a confiabilidade dos outros. Para Eddy, Gallup, e Povinelli (1993), o facto de sermos capazes de atribuir emoções humanas aos animais (antropomorfismo) teria permitido que conseguíssemos colocarmo-nos também no seu lugar.

No entanto, na literatura científica, a ligação entre o afeto por animais de estimação e o afeto por humanos não é consensual. Os estudos tanto defendem que as pessoas com grande afeto pelos animais também têm sentimentos calorosos para com humanos, como falham em encontrar uma relação (para mais informação, ver Paul, 2005, pp. 168). No entanto, no que toca à relação entre a empatia dirigida a humanos e animais, a literatura aponta para uma ligação positiva, embora fraca (Ellingsen, Zanella, Bjerkås, \& Indrebø, 2010; Emauz et al., 2016; Paul, 2000), sugerindo que diferentes fatores poderão estar a pesar de maneira distinta na forma de nos sentirmos afetados quando se trata de um conspecífico ou de um indivíduo doutra espécie.

\section{Fatores da Empatia para com Humanos}

Autores de diferentes áreas têm assinalado uma diversidade de fatores que afetam a empatia com humanos: fatores demográficos, como o sexo, a idade e a herança cultural; fatores intrínsecos, como a personalidade e predisposições genéticas de conduta; e, fatores ligados ao ambiente familiar, como o estilo de vinculação. Entre os fatores intrínsecos da empatia com humanos, incluem-se a capacidade de regular as próprias emoções (Eisenberg, 2000), a predisposição genética (Rodrigues, Slaslow, Garcia, John, \& Keltner, 2009), traços de personalidade (Kavanagh, Signal, \& Taylor, 2013), níveis de compaixão (Batson, Lishner, Cook, \& Sawyer 2005), bem como a perceção da semelhança com o outro (Davis, 1994). Por outro lado, fatores ligados ao ambiente familiar durante o desenvolvimento humano, como o tipo de vinculação com os progenitores (Schore, 2001), a qualidade do ambiente vivido em casa (Zahn-Waxler \& Radke-Yarrow, 1990) e o comportamento exibido pelos pais durante a infância do indivíduo (Hoffman, 1975) parecem influenciar o desenvolvimento da empatia. No âmbito deste estudo, iremos dar mais 
ênfase aos aspetos demográficos. Quer na empatia dirigida a humanos quer na dirigida a animais, as mulheres têm apresentado níveis mais elevados do que os homens (Apostol, Rebega, \& Miclea, 2013; Daily \& Morton, 2006; Mestre, Samper, Frías, \& Tur, 2009; O’Brien, Konrath, Grühn, \& Hagen, 2013; Paul, 2000; Toussaint \& Webber, 2005). Em termos da empatia emocional, as mulheres, quando comparadas com os homens, não só têm evidenciado uma maior resposta emocional à dor de outros, como maior eficácia no reconhecimento das emoções (Christov-Moore et al., 2014). Esta maior predisposição do sexo feminino para a empatia não é exclusiva dos humanos, estando presente noutras espécies, particularmente nas espécies altriciais, em que as crias dependem das mães durante um longo período pós-natal, sugerindo assim que a maior empatia apresentada pelo sexo feminino tenha uma componente mais biológica do que cultural (Christov-Moore et al., 2014).

Relativamente à idade, a empatia com humanos parece ter o seu auge em adultos numa fase média da vida (O’Brien et al., 2013), sendo que a diminuição da empatia na fase sénior está geralmente associada a fatores socioeconómicos como a viuvez e as limitações físicas e de saúde (Schieman \& Gundy, 2000). No estudo conduzido por Paul (2000), a vivência com crianças em casa estava associada a uma maior empatia dirigida a humanos. Este foi o único estudo em que encontramos esta relação entre empatia dirigida a humanos e o facto de ter crianças em casa.

No que concerne ao impacto da cultura na empatia com outros seres humanos, Trommsdorff, Friedlmeier e Mayer (2007), ao examinarem as respostas empáticas de crianças perante o desconforto de outras crianças, em dois países ocidentais e dois do sudeste asiático, descobriram que as crianças do ensino pré-escolar oriundas do sudeste asiático demonstravam mais desconforto pessoal e menos comportamentos de ajuda empática do que as crianças de origem ocidental. Cassels, Chan, Chung, e Birch (2010) procuraram as mesmas diferenças em adolescentes, e descobriram que o grupo ocidental reportava maiores níveis de preocupação empática e menos desconforto pessoal quando confrontado com o estado emocional negativo de uma outra pessoa. Estes resultados sugerem uma resposta emocional mais orientada para o outro, sendo esta, na cultura ocidental, associada a maiores níveis de comportamentos empáticos e de ajuda. Os autores salientam ainda a importância do papel dos pais no desenvolvimento da aprendizagem social e emocional das crianças.

\section{Fatores da Empatia para com Animais}

No que se refere aos preditores da empatia para com animais, foram até ao presente assinalados os fatores proximidade genética, vivência com animais de estimação e o sexo, que à semelhança da dirigida a humanos, também é mais elevada no sexo feminino (Paul, 2000; Rothberger \& Mican, 2014). Para o presente estudo, considerou-se relevante incluir os fatores associados às atitudes para com os animais mais referenciados na literatura, uma vez que o estudo das atitudes para com os animais é mais frequente e a sua literatura mais abundante. Embora se trate de conceitos distintos, a empatia dirigida a animais e as atitudes para com os animais encontram-se relacionadas (Ellingsen et al., 2010; Wagstaff, 1991) e podem apontar pistas para procurar fatores na origem da empatia com animais comuns à empatia dirigida a humanos e a membros de outras espécies.

Assim, entre os fatores que têm sido associados às atitudes para com os animais estão novamente $o$ sexo (Herzog, Betchart, \& Pittman, 1991; Mathews \& Herzog, 1997), onde são as mulheres quem mostra maior preocupação com os direitos e com o bem-estar animal (Broida Tingley, Kimball, \& Miele, 1993; Taylor \& Signal, 2005) e a vivência com animais de estimação (Ellingsen et al., 2010; Furnham, McManus, \& Scott, 2003; Paul, 2000; Paul \& Serpell, 1993). No plano das atitudes, são apontados ainda traços da personalidade como a agradabilidade, a extroversão (Broida et al.,1993; Furnham et al., 2003), o tipo de animal e o seu uso, com uma clara diferenciação entre as atitudes para com animais de estimação, de laboratório, ou as espécies cinegéticas (Knight \& Barnett, 2008; Wells \& Hepper, 1997), e também as crenças nas capacidades mentais dos animais (Apostol, Rebega, \& Miclea, 2013; Hills, 1995; Knight, Nunkoosing, Vrij, \& Cherryman, 2003), e o vegetarianismo (Broida et al., 1993; Furnham et al., 2003; Paul \& Serpell, 1993). Um aspeto clássico e marcante das atitudes em relação aos animais é a religião. Por exemplo, judeus e cristãos, têm em geral uma visão de que os humanos têm o domínio e tutela dos animais (Lindzey \& Clarke, 2004; Szűcs, Geers, Jezierski, Sossidou, \& Broom, 2012); no entanto, no seio da Igreja Anglicana surgiram (ainda no séc. XVIII) e um pouco mais tarde na Igreja Metodista, preocupações vincadas com "o tratamento misericordioso dos animais", "o pecado da crueldade com os animais" ou mesmo com o bem-estar dos animais de uma forma mais proativa. Esta preocupação mais marcada nas igrejas cristãs dissidentes (com origem no Reino Unido) do que na Igreja Católica-Romana, ou na Igreja Ortodoxa (judaica), envolve há mais de 150 anos a denúncia de práticas de exploração dos animais ou mesmo o questionamento da inclusão de carne na dieta (Kean, 1998). Já a maioria dos budistas e hindus, acredita na reincarnação, que se devem poupar e respeitar outras formas de vida, adotando entre práticas 
de tratamento não cruel, uma dieta que exclui animais e uma visão mais igualitária dos direitos de humanos e de outros animais (e.g. Szücs et al., 2012).

Para o efeito deste estudo, importa salientar alguns destes fatores. Por exemplo, a importância da vivência com animais de estimação durante a infância, que estará ligada a um incremento da empatia com os outros animais e não apenas com os de estimação (Paul \& Serpell, 1993; Rothberger \& Mican, 2014). A relação com animais de estimação durante a infância, que não só tem efeitos futuros ao nível de uma atitude mais positiva para com os animais e humanos, como tem sido associada a uma maior preocupação ética com a alimentação e a um maior envolvimento em associações de proteção animal e da natureza (Paul \& Serpell, 1993). 0 facto da vivência com animais de estimação, sobretudo durante o período da infância, ser preditor de empatia com animais é importante, pois sugere a necessidade de uma exposição bastante específica e precoce ao alvo da empatia para que esta possa mais tarde ser experimentada com esse alvo. Esta observação leva-nos a refletir acerca dos mecanismos subjacentes a tal especificidade:

a) processos semelhantes ao "imprinting", formulado por Konrad Lorenz em 1935 (MacFarland, 1987), e à vinculação (Bowlby, 1969) em que a exposição precoce dentro duma determinada janela temporal leva à inclusão do animal numa comunidade de iguais, num grupo social relevante do ponto de vista emocional. Com estes mecanismos, tornar-se-ia compreensível que a empatia com animais fosse, em regra, inferior à empatia com humanos, atendendo a que a exposição a humanos é sempre superior, mas excetuar-se-iam algumas espécies (por exemplo, animais domésticos com que se tivesse tido grande proximidade em criança);

b) uma oportunidade privilegiada de interação com animais, conducente à perceção dos mesmos como seres sencientes, dotados de emoções e vidas complexas num período de desenvolvimento, como é o que se estende até à adolescência, em que as crianças adquirem muitas competências de reconhecimento das emoções (Harris, 2000);

c) um processo de modelagem social, como os assinalados por Bandura e colegas (Bandura, Ross, \& Ross, 1961) ou por Hoffman (1975), em que as atitudes e comportamentos dos pais/outros adultos vistos como modelos passam a integrar os esquemas de interação com o alvo animal.

Outro aspeto que tem sido mencionado frequentemente como associado à empatia e às atitudes para com os animais, é o tipo de alimentação, nomeadamente as escolhas de uma dieta baseadas na ética e no bem-estar animal. Um estudo conduzido por Filippi et al. (2010) comparou as diferenças nas respostas neuronais de participantes com regimes alimentares distintos (vegetarianos, veganos e omnívoros) quando observavam imagens de animais e humanos em sofrimento. Neste estudo, vegetarianos e veganos mostraram um maior recrutamento das áreas relacionadas com a empatia (nomeadamente o córtex cingulado anterior e o giro frontal inferior), não só quando observavam imagens negativas envolvendo animais, mas também humanos. Em suma, este estudo sugere que indivíduos que optam por um regime alimentar baseado na preocupação ética para com os animais apresentam padrões de resposta emocional que a nível neuronal pressupõem uma maior empatia.

Até ao presente momento não foram encontrados estudos específicos sobre diferenças culturais na empatia dirigida a animais. No entanto, a relação entre humanos e os seus animais de estimação tem sofrido grandes mudanças ao longo dos tempos em diferentes países onde alguns animais se tornaram populares como animais de estimação, como é o caso dos coelhos no Japão, onde estão a deixar de ser vistos como comida para passar a ser fiéis companheiros, como sucede na China, no Vietname ou na Tailândia (ver Pręgowski, 2016). Da mesma forma que assistimos a mudanças na forma como são tratados os animais em vários países, também podemos situar, num plano histórico, eventos e tendências relevantes nos povos anglo-saxónicos no que concerne à sua sensibilidade e preocupação com os animais. Para começar, considera-se que o movimento para os direitos dos animais teve origem no Reino Unido, tendo sido neste país que foi fundada, em 1840, a primeira sociedade de proteção dos animais (a RSPCARoyal Society for the Prevention of Cruelty to Animals), ainda hoje muito ativa, sucedida de uma sociedade quase gémea - a SPCA, Society for the Prevention of Cruelty to Animals - em S. Francisco nos EUA, em 1860. Foi também no Reino Unido que surgiu, em 1944, a primeira sociedade vegana e onde foi publicado o primeiro manifesto explícito dos direitos dos animais pelo filósofo britânico Tom Reagan (1983). Aquela que é atualmente a maior e mais influente associação de defesa dos direitos dos animais e do bem-estar animal - a PETA, People for the Ethical Treatment of Animals - nasceu nos EUA em 1993 (www.peta.org), onde se encontra ainda a sua sede, apesar dos vários polos mundiais. Não pretendendo sermos exaustivos neste argumento, julgamos que existe ampla evidência histórica mostrando que no Reino Unido e nos Estados Unidos da América existem elementos culturais que favorecem diferenças no que concerne à preocupação com os outros animais, e por conseguinte, à empatia com o seu sofrimento e com as suas emoções. 


\section{Objetivos do estudo}

No presente estudo pretende-se investigar a relação entre a empatia dirigida a humanos e a outros animais, explorando alguns fatores que poderão predizer as duas formas de empatia. Destacando dos fatores acima assinalados, o sexo e a vivência com animais de estimação tanto no passado como no presente (Paul \& Serpell, 1993; Paul 2000; Furnham et al, 2003; Ellingsen et al., 2010), procuramos explorar também como potenciais preditores da empatia pelos animais, a pertença ou envolvimento numa associação ou Organização Não Governamental (ONG) dirigida a animais e, também, a dieta alimentar (vegetarianismo/veganismo). No que concerne à empatia para com humanos, também investigámos o envolvimento em ONGs humanitárias, e ter ou não filhos, como preditores da empatia dirigida a humanos. A avaliação de aspetos potencialmente culturais foi feita através de um inquérito online em duas línguas (portuguesa e inglesa) tendo como alvos uma população anglo-saxónica e uma população de raiz lusófona e maioritariamente portuguesa.

\section{MÉTODO}

\section{Participantes}

O grupo de língua portuguesa distribuiu-se se por $87.3 \%$ de portugueses e $8.8 \%$ de brasileiros $(3.9 \%$ sem resposta). Neste grupo, observou-se uma taxa de desistência de $36 \%$, obtendo-se um total de 223 participantes, dos quais $190(85.2 \%)$ do sexo feminino e 33 (14.8\%) do sexo masculino. A idade foi medida através de uma variável categórica quantificada em cinco níveis: $\leq 18$ anos (1.3\%), 19-24 anos (6.7\%), 25-34 anos (39.9\%), 35-44 anos (33.2\%), $\geq 45$ anos (18.8\%).

o grupo que respondeu em língua inglesa distribuiu-se em $62.1 \%$ participantes norte americanos, $13.7 \%$ europeus, $11.1 \%$ australianos, $2.6 \%$ africanos, $2 \%$ asiáticos, $0.7 \%$ da América do Sul e $7.8 \%$ semresposta quanto à origem. Neste grupo observou-se uma taxa de desistência de $53 \%$, obtendo-se dados de um total de 154 participantes, dos quais 134 (87\%) do sexo feminino e 19 (12.3\%) do sexo masculino (uma sem resposta). Tal como na amostra portuguesa, a idade foi representada por uma variável categórica quantificada nos mesmos cinco níveis. Para efeitos da análise de preditores na população anglo saxónica, foram usados apenas os dados dos participantes dos Estados Unidos e do Reino Unido, num total de 109 participantes, distribuídos por $88.1 \%$ americanos e $11.9 \%$ do Reino Unido. A distribuição da percentagem relativamente ao sexo nesta nova amostra manteve-se relativamente semelhante, 95 $(87.2 \%)$ do sexo feminino e $14(12.8 \%)$ do sexo masculino.

\section{Procedimento}

Os participantes foram recrutados através de e-mails e de links em páginas institucionais, do facebook e do twitter, a um blog que foi criado com informações sobre o estudo. Foi igualmente feito um pedido de colaboração na disseminação do estudo e respetivo link, através de mensagem privada às páginas de associações de proteção animal, bem como a instituições de carácter humanitário (dirigido a humanos), tanto nacionais como internacionais. O blog, bilingue (português e inglês) ligava-se a um questionário online, disponível nas duas línguas e gerado com o software Qualtrics (versão 2014). Os dados foram extraídos das respostas obtidas entre 20 de Fevereiro e 20 de Maio de 2014.

\section{Instrumentos}

\section{Escala de empatia para com animais (EEA/AES)}

Para medir a empatia para com os animais junto da população anglo-saxónica, utilizámos a escala originalmente desenvolvida por Paul (2000), a Animal Empathy Scale (AES). Esta escala foi desenvolvida a partir de uma escala que mede a empatia emocional dirigida a humanos (QMEE - Mehrabian \& Epstein, 1972), e reestruturada por Paul (2000) com novos itens baseados em respostas e afirmações dadas em entrevistas a estudantes e membros do público em geral, no sentido de incluir apreciações sobre a forma como os animais eram tratados. A escala é constituída por 22 itens, metade dos quais representam sentimentos de empatia e a outra metade, sentimentos contrários. Itens como "Fico perturbado(a) ao ver um animal a sofrer" representam sentimentos de preocupação pelos animais, enquanto que itens como "As pessoas geralmente exageram as emoções e sentimentos que atribuem aos animais" representam um desconforto ou incómodo do próprio face aos comportamentos dos outros para com os animais. As respostas são dadas através de uma escala do tipo Likert com nove pontos, que variam desde "Discordo muitíssimo" até "Concordo muitíssimo". Os 11 itens que contêm sentimentos opostos aos empáticos são cotados inversamente de forma a permitir que uma pontuação mais elevada da AES corresponda a um maior nível de empatia. 
A Escala de Empatia com Animais (EAA), que consiste numa versão da AES, adaptada para português por Emauz et al. (2016) foi a usada para a avaliação da empatia com animais, na versão do inquérito em língua portuguesa. Esta versão é composta por 13 itens, resultantes de uma análise fatorial exploratória e de uma confirmatória que revelaram a existência de duas componentes, Ligação Emocional com Animais (LEA) e Preocupação Empática com os Animais (PEA). A LEA é composta por sete itens que representam uma resposta emocional à forma como as pessoas vêm os animais, formulados na negativa $\mathrm{e}$ por isso cotados inversamente, como por exemplo o item "Há muitas pessoas que são exageradamente afetuosas com os seus animais de estimação". A subescala PEA é composta por seis itens, que na sua maioria refletem sentimentos de preocupação com os animais, tais como "Fico indignado(a) ao ver animais a serem maltratados". A EEA, embora com menos itens que a escala inglesa original (AES), apoiase em boas características psicométricas (Emauz et al., 2016).

\section{Índice de Reatividade Interpessoal (IRI)}

Para medir a empatia dirigida a humanos, utilizou-se o Índice de Reatividade Interpessoal (IRI; Davis, 1980). Este índice inclui quatro dimensões da empatia, medidas através de 28 itens divididos pelas subescalas Tomada de Perspetiva, Fantasia, Desconforto Pessoal e Preocupação Empática. As duas primeiras representam o lado mais cognitivo e as duas últimas a faceta mais emocional. A Tomada de Perspetiva representa a capacidade de nos colocarmos no lugar de outro, como é exemplo o item "Fico muitas vezes emocionado/emocionada com coisas que vejo acontecer". A subescala Fantasia mede a capacidade de nos transportarmos de forma imaginária para situações fictícias, como por exemplo o item "Facilmente me deixo envolver nos sentimentos das personagens de um romance". O Desconforto Pessoal é uma subescala que mede respostas mais orientadas para o próprio, refletindo sentimentos de medo, apreensão e desconforto, por exemplo "Em situações de emergência, sinto-me desconfortável e apreensivo/apreensiva". Por fim, a subescala Preocupação Empática reflete sentimentos calorosos, de compaixão e preocupação para com os outros, como é exemplo o item "Tenho muitas vezes sentimentos de ternura e preocupação pelas pessoas menos afortunadas do que eu". Neste estudo, na versão portuguesa do inquérito, foi utilizada a versão de Limpo, Alves e Castro (2010), que contém menos quatro itens do que a versão original de Davis (1980), mas mantém a estrutura multidimensional da empatia representada pelas quatro subescalas acima referidas. A estrutura desta versão portuguesa apresenta um modelo com boa fiabilidade e bons índices de consistência interna tornando-a adequada para este estudo.

Finalmente, o inquérito incluiu questões de carácter demográfico (sexo e idade), envolvimento com ONGs, dieta alimentar e ter ou não filhos e religião (onde os participantes podiam optar pelas seguintes categorias: cristianismo, hinduísmo, budismo, islamismo, sem religião, outra, e deixar especificações, como por exemplo, ser católico ou protestante), exatamente na mesma ordem, em ambas as versões de língua portuguesa e inglesa. No caso da questão relativa à dieta alimentar, o inquérito oferecia cinco opções de regimes alimentares (regular, regular sem carne, vegetariano, vegano, outro). No entanto, optou-se por colapsar os resultados em apenas duas escolhas (omnívoro e vegano/vegetariano) por haver poucas respostas por categoria e a religião foi eliminada das análises por ser muito homogénea em ambas as amostras, onde todos os respondentes que não deixaram a resposta em branco ou responderam "outra", eram cristãos.

\section{RESULTADOS}

\section{Relação entre Empatia com Animais e com Humanos}

No grupo de língua portuguesa, a empatia com humanos e com outros animais, medidas respetivamente através da EEA e do IRI, mostraram-se positivamente correlacionadas. Embora significativa, a correlação foi fraca, $r(221)=.19, p<.01$.

No grupo de língua inglesa, a empatia dirigida a animais e a humanos revelaram-se igualmente correlacionadas de forma significativa e positiva, com uma correlação igualmente fraca, $r(152)=.18, p<$ .059 .

\section{Preditores de Empatia com Humanos e com Outros Animais}

A estatística descritiva (Tabela 1) mostra que, em geral, o sexo feminino apresenta maior empatia com animais e com humanos, embora a diferença entre os sexos não seja significativa no grupo anglo-saxónico no que concerne à empatia dirigida a humanos.

Verificou-se, nos respondentes lusófonos, que a empatia com animais foi mais elevada nas mulheres $(p=.002)$, nas pessoas que vivem com animais de estimação $(p=.001)$, nas pessoas ligadas a uma ONG, enquanto voluntárias ou associadas $(p=.007)$ e nos participantes que não têm filhos $(p=.029)$. 
Na amostra anglo-saxónica, a empatia com animais também foi significativamente mais elevada nos participantes do sexo feminino $(p=.039)$, nas pessoas não ligadas a ONGs $(p=.013)$, nos participantes vegetarianos/veganos $(p<.001)$; nos que vivem com animais de estimação é mais elevada, embora esta diferença não chegasse a atingir significância $(p=.215)$.

Tabela 1. Análise descritiva das médias das variáveis preditoras da empatia com humanos (medida através do IRI) e da empatia com animais (medida através da EEA/AES) nos grupos lusófono e anglosaxónico.

\begin{tabular}{|c|c|c|c|c|c|c|c|c|}
\hline \multirow[b]{3}{*}{ Preditores } & \multicolumn{4}{|c|}{ Lusófonos } & \multicolumn{4}{|c|}{ Anglo-saxónicos } \\
\hline & \multicolumn{2}{|c|}{$\begin{array}{c}\text { Empatia com } \\
\text { animais }\end{array}$} & \multicolumn{2}{|c|}{$\begin{array}{c}\text { Empatia com } \\
\text { humanos }\end{array}$} & \multicolumn{2}{|c|}{$\begin{array}{c}\text { Empatia com } \\
\text { animais }\end{array}$} & \multicolumn{2}{|c|}{$\begin{array}{c}\text { Empatia com } \\
\text { humanos }\end{array}$} \\
\hline & $M$ & $D P$ & $M$ & $D P$ & $M$ & $D P$ & $M$ & $D P$ \\
\hline Mulheres & 6.06 & 0.67 & 3.55 & 0.37 & 5.94 & 0.58 & 4.41 & 0.56 \\
\hline Homens & 5.53 & 0.85 & 3.33 & 0.35 & 5.56 & 0.87 & 4.30 & 0.30 \\
\hline Animais estimação & 6.09 & 0.68 & 3.51 & 0.38 & 5.92 & 0.59 & 4.42 & 0.56 \\
\hline Sem animais de estimação & 5.66 & 0.77 & 3.51 & 0.37 & 5.70 & 0.83 & 4.29 & 0.25 \\
\hline Ter filhos & 5.77 & 0.82 & 3.48 & 0.40 & 5.78 & 0.72 & 4.44 & 0.47 \\
\hline Não ter filhos & 6.09 & 0.64 & 3.53 & 0.36 & 5.97 & 0.55 & 4.38 & 0.58 \\
\hline Ligação a ONG & 6.33 & 0.47 & 3.54 & 0.34 & 5.72 & 0.73 & 4.38 & 0.62 \\
\hline Sem ligação a ONG & 5.79 & 0.77 & 3.50 & 0.39 & 6.03 & 0.50 & 4.41 & 0.46 \\
\hline Vegetarianos & 6.27 & 0.57 & 3.59 & 0.30 & 6.12 & 0.51 & 4.41 & 0.59 \\
\hline Omnívoros & 5.95 & 0.74 & 3.51 & 0.38 & 5.55 & 0.64 & 4.39 & 0.45 \\
\hline
\end{tabular}

Realizaram-se análises de regressão linear múltiplas para os dados de cada uma das versões/populações alvo (lusófona e anglo-saxónica), examinando os efeitos do sexo, vivência com animais de estimação, ligação a uma ONG, vegetarianismo e ter filhos na empatia dirigida a humanos e outros animais. Tratando-se de variáveis binárias, foram codificadas como $0=$ homens, $1=$ mulheres; viver com animais de estimação $=1$, não viver $=0$; estar ligado a uma $0 N G=1$, não estar $=0$; e ter filhos $=1$, não ter filhos $=0$; ser vegetariano/vegano $=1$, não ser vegetariano/vegano $=0$.

$\mathrm{Na}$ análise de regressão múltipla para a amostra de língua portuguesa, não se incluiu a variável regime alimentar (vegetariano/vegano ou omnívoro) porque, tal como se pode ver na Tabela 2 , mais de 90\% dos inquiridos tinham uma alimentação omnívora.

Tabela 2. Frequência e percentagem das variáveis preditoras nos grupos lusófono e anglo-saxónico.

\begin{tabular}{|c|c|c|c|c|}
\hline \multirow[b]{2}{*}{ Variáveis preditoras } & \multicolumn{2}{|c|}{ Lusófonos } & \multicolumn{2}{|c|}{ Anglo-saxónicos } \\
\hline & Frequência & Percentagem & Frequência & Percentagem \\
\hline Mulheres & 192 & $84.20 \%$ & 95 & $87.00 \%$ \\
\hline Homens & 36 & $15.80 \%$ & 14 & $12.80 \%$ \\
\hline Ter animais de estimação & 168 & $73.70 \%$ & 96 & $88.10 \%$ \\
\hline Não ter animais de estimação & 57 & $25.00 \%$ & 13 & $11.90 \%$ \\
\hline Ter filhos & 80 & $35.10 \%$ & 44 & $40.40 \%$ \\
\hline Não ter filhos & 146 & $64.00 \%$ & 65 & $59.60 \%$ \\
\hline Ligação a ONG & 81 & $35.50 \%$ & 47 & $43.10 \%$ \\
\hline Sem ligação a ONG & 144 & $63.20 \%$ & 62 & $56.90 \%$ \\
\hline Vegetarianos/veganos & 19 & $8.30 \%$ & 65 & $59.60 \%$ \\
\hline Omnívoros & 207 & $90.80 \%$ & 42 & $38.50 \%$ \\
\hline
\end{tabular}

Da testagem do modelo incluindo todas estas variáveis na predição da empatia com animais, resultou, na amostra de língua portuguesa, uma equação de regressão significativa, $F(4,210)=12.61, p$ $<.001$, com um tamanho de efeito pequeno $\left(f^{2}=.23\right)$, explicando quase $20 \%$ da variância $\left(R^{2}=.19\right)$. Como pode ser consultado na Tabela 3 , as variáveis sexo $(p=.01)$, ter animais de estimação $(p=.03)$, estar ligado a uma ONG ( $p=.01)$ e não ter filhos $(p=.04)$ são preditoras significativas da empatia com animais. 
Tabela 3. Resumo da análise de regressão para as variáveis preditoras da empatia com animais (medida através da EEA) no grupo lusófono.

\begin{tabular}{lccc}
\hline & & Empatia com Animais (EEA) \\
\cline { 2 - 4 } Variável & $\boldsymbol{B}$ & \multicolumn{1}{c}{$\boldsymbol{S E}$} & \multicolumn{1}{c}{$\boldsymbol{\beta}$} \\
\hline Constante & 70.40 & 1.78 & $0.16^{*}$ \\
Sexo & 4.14 & 1.65 & $0.20^{* *}$ \\
Animais estimação & 4.15 & 1.34 & $0.25^{* *}$ \\
ONG & 4.74 & 1.20 & $-0.17^{* *}$ \\
Filhos & -3.35 & 1.21 & \\
\hline
\end{tabular}

Nota: ${ }^{*} p<.05,{ }^{* *} p<.01$

Quanto à empatia para com humanos, medida através do IRI, de todas as variáveis preditoras, apenas o sexo foi significativo $(p=.01)$.

No grupo que respondeu ao questionário em língua inglesa, optámos por realizar a análise dos modelos preditivos da empatia com animais e com humanos, apenas com os participantes americanos e do Reino Unido, que pelas razões histórico-culturais apontadas na introdução, constituem, do ponto de vista da preocupação com o sofrimento e bem-estar dos animais, uma população relativamente homogénea. As análises incluíram as variáveis: sexo, vivência com animais de estimação, pertença a uma ONG, ter filhos e regime alimentar (vegetariano/vegano ou omnívoro).

A testagem do modelo, incluindo as variáveis acima descritas, na predição da empatia com animais, na amostra de língua inglesa, resultou numa equação de regressão significativa $F(5,101)=7.03, p<.001$, sendo o modelo apresentado, explicativo de $26 \%$ da variância $\left(R^{2}=.26\right)$ com um tamanho de efeito médio $\left(f^{2}=.35\right)$. Como pode ser observado na Tabela 4 , os resultados mostraram que as variáveis sexo $(p=.04)$, ter animais de estimação $(p=.05)$, e ser vegetariano/vegano $(p=.01)$ são preditoras significativas da empatia com animais. No entanto, nenhuma das variáveis do modelo é preditora da empatia dirigida a humanos $(p=.41)$.

Tabela 4. Resumo da análise de regressão para as variáveis preditoras da empatia com animais (medida através da AES) no grupo anglo-saxónico.

\begin{tabular}{lccc}
\hline & & Empatia com Animais (AES) \\
\cline { 2 - 4 } Variável & $\boldsymbol{B}$ & $\boldsymbol{S E} \boldsymbol{B}$ & \multicolumn{2}{c}{$\boldsymbol{\beta}$} \\
\hline Constante & 111.78 & 3.18 & $0.18^{*}$ \\
Sexo & 7.41 & 3.65 & $0.17^{*}$ \\
Animais estimação & 7.22 & 2.86 & -0.03 \\
ONG & -0.94 & 2.47 & -0.12 \\
Filhos & -3.27 & 2.91 & $0.40^{* *}$ \\
Vegetariano/vegano & 11.16 & & \\
\hline
\end{tabular}

Nota: ${ }^{*} p<.05,{ }^{* *} p<.01$

\section{DISCUSSÃO}

O presente estudo teve como objetivo explorar possíveis variáveis preditoras da empatia e averiguar se os fatores que predizem empatia dirigida a humanos são os mesmos que predizem a empatia dirigida a outros animais. Por outro lado, pretendeu-se apurar se estes fatores são transversais a pessoas originárias de diferentes culturas (lusófona e anglo-saxónica), que embora possam ser de locais geográficos diferentes, partilham, de certa forma, aspetos culturais comuns.

Contrariando a expectativa baseada na presunção de origem comum da empatia dirigida a humanos e a outros animais, ancorada no sistema de cuidados maternos (de Waal, 2008; Paul, 2000), atualmente com forte aceitação (Gaspar, 2014), a correlação verificada entre as duas formas de empatia foi, no presente estudo e em ambos os grupos (de língua portuguesa e anglo-saxónicos), fraca. Veio, no entanto, a sublinhar a tendência de alguns estudos anteriores (Emauz et al., 2016; Paul, 2000). Esta observação, apesar de compatível com a existência de uma base comum às duas formas de empatia, sugere fortemente a existência de fatores distintos subjacentes ao desenvolvimento de cada uma.

Com vista à compreensão dos fatores preditores da empatia dirigida a humanos e da empatia dirigida a outros animais abordamos, seguidamente, os resultados de cada um daqueles que neste estudo foram explorados: sexo, a vivência com animais de estimação, ligação a uma ONG, tipo de regime alimentar e ter ou não filhos.

Tanto o sexo feminino como a vivência com animais de estimação foram preditores da empatia dirigida a animais (em ambas as amostras), enquanto que o sexo feminino surgiu como único preditor da 
empatia dirigida a humanos, e apenas na amostra de língua portuguesa. Adicionalmente, verificou-se que havia diferenças entre as duas amostras de língua portuguesa e anglo-saxónica, nomeadamente nas variáveis que prediziam a empatia dirigida a animais. Na amostra lusófona, estar associado a uma ONG de cariz humanitário (dirigida a humanos e/ou animais) mostrou-se um bom preditor da empatia dirigida a animais, enquanto que na amostra anglo-saxónica a dieta alimentar foi o preditor com mais peso.

A vivência com animais de estimação é um dos fatores apontados na literatura como preditora de empatia ou atitudes para com animais, sobretudo se esta tiver tido lugar ou início na infância (Paul 2000). É possível que esta relação se deva ao facto de nessa altura se estabelecer uma forte vinculação com os animais da casa, o que parece ser prevalente nos lares com animais do Reino Unido (e.g. Hawkins, Dawkins, \& Scottish SPCA, 2017). Segundo Melson (2003), os lares onde existem muitos animais de estimação providenciam uma maior oportunidade para a interação, cuidados e afeto, que são por sua vez vistos como precursores da empatia. A importância dum processo de ligação emocional precoce poderá ter sido parcialmente validada num estudo recente (Rothberger \& Mican, 2014), em que os investigadores exploraram a ligação entre ter tido um animal de estimação na infância, a sua vinculação a um animal em particular nesse período, a empatia-traço na vida adulta, a visão dos animais como mais parecidos emocionalmente com os humanos e a adoção de comportamentos generalizados de proteção dos animais, como o vegetarianismo. Verificaram que apesar da vinculação aos animais na infância ser preditora da empatia com animais na vida adulta, os efeitos da exposição na infância eram não só menores que os da empatia, como a adoção destes comportamentos protetores dos animais nos adultos, era mediada pela empatia com animais. Assim, a empatia surge como causa imediata, mas a exposição aos animais, e em particular a vinculação aos mesmos, revela-se como uma experiência precoce importante na construção dessa empatia.

Relativamente à ligação a uma ONG e ao tipo de regime alimentar, observaram-se diferenças sobretudo entre a amostra de língua portuguesa e a anglo-saxónica. Na primeira, estar ligado a uma ONG, em particular de proteção animal, tanto através do associativismo como do voluntariado, revelou-se um preditor significativo na empatia para com animais. Na amostra anglo-saxónica, maioritariamente composta por pessoas dos Estados Unidos da América, a ligação a uma ONG não foi um preditor significativo da empatia com animais, sendo a variável com mais peso, o seguimento de uma dieta vegetariana/vegana. Fazer voluntariado numa associação, ou ONG, é uma ação que pode envolver tempo e/ou dinheiro, e que visa sobretudo beneficiar outras pessoas, causas ou grupos. As razões que levam ao voluntariado são variadas, mas os estudos existentes sugerem que o voluntariado se baseia sobretudo nos valores, ou seja, na genuína vontade de ajudar os outros (Akintola, 2011; Clary \& Snyder, 1999). Temse verificado que os países em que há maior adesão ao associativismo/voluntariado são aqueles que apresentam um melhor desenvolvimento económico (Aydinli, Bender, \& Chasiotis, 2013), o que é consistente com os resultados deste estudo, onde se verificou um envolvimento mais elevado do grupo anglo-saxónico em ONGs do que o de língua portuguesa, reforçando a ideia de que os recursos podem ser um fator relevante. No entanto, são os portugueses ligados a associações e ONGs de proteção animal que apresentam os maiores níveis de empatia para com animais. Poderão ser as pessoas com maiores recursos a estar mais disponíveis para esta forma de ajuda, mas para explorar esta questão será importante averiguar se existem outras variáveis que associem a maior participação (ativa) via ONGs e informação, como a sensibilização para as causas que se procuram apoiar, o estatuto socio-cultural, níveis de literacia, ou ainda oportunidades de exposição a estas causas e às respetivas formas de resposta, e se os recursos ou o estatuto socio-cultural poderão de alguma forma ser fatores latentes aos restantes. 0 facto de o vegetarianismo surgir como preditor da empatia com animais no grupo anglo-saxónico, é consistente com o bem-estar animal, sendo uma das duas principais razões invocadas para as pessoas se tornarem vegetarianas (Fox \& Ward, 2008). Nos Estados Unidos, um estudo intitulado "Vegetarianism in America" e publicado pela Vegetarian Times (vegetariantimes.com) mostrou que 7.3 milhões de americanos adultos (3.2\% da população) seguem uma dieta vegetariana, dos quais 1 milhão (cerca de $0.5 \%$ ) são veganos. 0 veganismo sendo, mais do que uma dieta, uma opção de consumo de produtos (alimentares, domésticos, vestuário, etc.) que não impliquem a morte ou o sofrimento de animais, está claramente relacionado com as atitudes face aos mesmos e poderia ter sido, se isolado, um preditor mais robusto da empatia com animais. Em Portugal, o único estudo feito sobre o número de vegetarianos foi realizado pela Nielsen (2007) para o Centro Vegetariano, estimando que cerca 30.000 portugueses eram vegetarianos, ou seja, cerca de $0.3 \%$ da população. Alguns estudos recentes trazem pistas acerca da ligação do vegetarianismo à empatia: esta relação poderá ser mediada pela quantidade de informação disponível, como sugerem dados da Human League e da Vegan Outreach (Singer, 2015) resultantes da contabilização do número de novos vegetarianos por vídeo informativo visto, e por custos de divulgação (sob a forma de folhetos, sites, etc), sendo a informação contida naquele material relativa à natureza dos animais e ao sofrimento dos mesmos nos processos conducentes à alimentação humana (Singer, 2015). 
Adicionalmente, informação sobre o comportamento e a vida dos animais é um preditor importante da capacidade das crianças identificarem emoções em animais (Rocha, Gaspar \& Esteves, 2016), o que sugere a importância da informação disponível na componente cognitiva da empatia.

Relativamente às diferenças encontradas entre os sexos na empatia, os resultados foram de certo modo surpreendentes ao mostrarem que este efeito na empatia com humanos se verifica apenas no grupo de língua portuguesa. As mulheres têm tradicionalmente apresentado uma maior empatia e preocupação tanto pelos humanos como por animais, estando desde o séc. XIX mais ligadas a movimentos e associações de cariz humanitário do que os homens, sendo a diferença entre o envolvimento dos sexos em ONGs ainda mais marcada quando se trata de movimentos de defesa dos animais (e.g. Herzog, 2007; Herzog et al., 1991; Signal \& Taylor, 2006). 0 facto de no grupo anglo-saxónico o sexo não ser um preditor da empatia para com humanos, contradizendo a maioria dos estudos, foi de certo modo inesperado, mas não sem precedentes: por exemplo, num estudo de respostas empáticas a maus tratos perpetrados a humanos (homens e mulheres) e a animais (cães e gatos), as mulheres foram significativamente mais empáticas do que os homens com os animais, mas na empatia com humanos não se verificaram diferenças entre os sexos (Angantyr, Eklund, \& Hansen, 2011). É importante, contudo, ter em atenção o desequilíbrio entre o número de participantes do sexo masculino e do sexo feminino, ou seja, tanto na amostra de língua portuguesa como na amostra em língua inglesa os participantes masculinos foram bastante menos.

Paul (2000) havia encontrado uma correlação entre a empatia por humanos e o ter filhos, correlação essa que não foi encontrada neste estudo em nenhuma das duas amostras. Na amostra portuguesa foi inclusivamente encontrada uma correlação negativa entre o ter filhos e a empatia por animais. É possível que as pessoas que não tenham filhos redirecionem a sua atenção e afeto para os seus animais de estimação, acabando por apresentar níveis de empatia pelos animais acima da média. Um estudo recente (Nagasawa, Kikusui, Onaka, \& Ohta, 2009) veio mostrar que o tipo de resposta afetiva sentida quando olhamos fixamente para os nossos cães é semelhante à sentida quando olhamos para as nossas crianças, uma vez que é ativado o mesmo processo que conduz à libertação de oxitocina no cérebro, responsável pela sensação de amor e de ligação com o outro; acresce que os cães também libertam oxitocina quando olham fixamente para os seus tutores humanos, sendo, até ao momento, a única espécie relativamente à qual existem dados neste sentido (a experiência repetida com lobos domesticados, não replicou o efeito).

\section{Limitações e Investigação Futura}

Os resultados do presente estudo não permitem elucidar qual dos mecanismos mencionados na introdução (imprinting, modelagem social ou interação com animais na infância) prevalece, ou mesmo se todos terão alguma contribuição, pois, com a informação disponível (i.e., sabendo apenas se em algum momento da sua vida, incluindo o presente, as pessoas tiveram animais de estimação) apenas nos permite sugerir que a experiência da interação com animais durante o período da infância, é o mais plausível. A este nível, seria útil futuramente comparar a empatia com animais em quem foi exposto à convivência com animais de estimação na infância com a de quem teve uma exposição equivalente iniciada apenas na vida adulta. Outra abordagem elucidativa seria longitudinal, avaliando possíveis alterações nas medidas de empatia dirigida a animais e a humanos em crianças e adolescentes com e sem animais de estimação desde a primeira infância. É profundamente importante, a nosso ver, compreender o que leva as pessoas com elevados níveis de empatia com animais, a desenvolverem, em diferentes países, formas distintas de atuar relativamente ao seu objeto de empatia. Futuramente seria interessante explorar o que tem maior impacto - se recursos, se facilitadores sociais ou a perceção de eficácia das várias formas de atuar no sentido de melhorar a vida dos animais, ou não contribuir para o seu sofrimento.

0 estudo também mostra claramente a necessidade de explorar fatores associados à empatia com humanos, tais como a personalidade ou o ambiente vivido em casa, que não foram explorados neste trabalho. Finalmente, as nossas amostras também constituem limitações em si mesmas, uma vez que incluem respondentes muito orientados para o bem-estar dos animais (dado que as ONGs colaboraram na divulgação do estudo), ou seja, poderão estar enviesadas relativamente à empatia com animais, e mesmo em relação à empatia com humanos, não sendo representativas da população em geral. Por outro lado, as amostras são limitantes, por não terem uma dimensão suficientemente grande em cada língua para ter sido feita uma análise mais fina, por países, o que teria permitido abordar questões culturais com maior rigor. Por exemplo, seria interessante explorar diferenças religiosas associadas a diferentes normas morais, mais ou menos abertas a novas ideias sobre a forma como vemos a sociedade, ou também comparar culturas mais individualistas com outras de tradição mais coletivista. Assim, em futuros estudos poder-se-ão alargar estas amostras, divulgando os inquéritos através de redes sociais mais abrangentes, para assim explorar de forma sistemática diferenças culturais relevantes. 
Apesar das limitações deste estudo exploratório, os seus resultados reforçaram o valor de alguns dos fatores estudados anteriormente como preditores da empatia dirigida a animais, como o sexo e a vivência com animais de estimação, acrescentando outros preditores como a ligação a uma ONG e o tipo de alimentação, cuja relação com a empatia com animais poderá futuramente ser mais aprofundada (por exemplo, incluindo possíveis fatores subjacentes a estas variáveis preditoras, como a motivação, informação, experiências e oportunidades).

\section{REFERÊNCIAS}

Akintola, O. (2011). What motivates people to volunteer? The case of volunteer AIDS caregivers in faithbased organizations in KwaZulu-Natal, South Africa. Health, Policy and Planning, 26(1), 53-62. http://dx.doi.org/10.1093/heapol/czq019

American Psychiatric Association. (2013). Diagnostic and statistical manual of mental disorders (5th ed.). Washington, DC.

Angantyr, M., Eklund, J., \& Hansen, E. M. (2011). A Comparison of empathy for humans and empathy for animals. Anthrozoos: A Multidisciplinary Journal of The Interactions of People \& Animals, 24(4), 369377. http://dx.doi.org/10.2752/175303711X13159027359764

Apostol, L., Rebega, O. L., \& Miclea, M. (2013). Psychological and socio-demographic predictors of attitudes toward animals. Procedia - Social and Behavioral Sciences, 78, 521-525. http://dx.doi.org/10.1016/j.sbspro.2013.04.343

Ascione, F. R. (1992). Enhancing children's attitudes about the humane treatment of animals: Generalization to human-directed empathy. Anthrozoös, 5(3), 176-191. http://dx.doi.org/10.2752/08927939278701142

Ascione, F. R., \& Weber, C. V. (1996). Children`s attitudes about humane treatment of animals and empathy: One-year follow up of a school-based intervention. Anthrozoös, 9(4), 188-195. http://dx.doi.org/10.2752/089279396787001455

Ascione, F. R., Weber, C. V., \& Wood, D. S. (1997). The abuse of animals and domestic violence: A national survey of shelters for women who are battered. Society \& Animals, 5(3), 205-218. http://dx.doi.org/10.1163/156853097X00132

Aydinli, A., Bender, M., \& Chasiotis, A. (2013). Helping and volunteering across cultures: Determinants of prosocial behavior. Online Readings in Psychology and Culture, 5(3). http://dx.doi.org/10.9707/2307-0919.1118

Bandura, A., Ross, D., \& Ross, S.A. (1961). Transmission of aggression through imitation of aggressive models. Journal of Abnormal and Social Psychology, 63, 575-582. http://dx.doi.org/10.1037/h0045925

Batson, C. D., Lishner, D. A., Cook, J., \& Sawyer, S. (2005). Similarity and nurturance : Two possible sources of empathy for strangers. Basic and Applied Social Psychology, 27(1), 15-25. http://dx.doi.org/10.1207/s15324834basp2701_2

Bowlby, J. (1969). Attachment and loss, Vol. 1: Attachment. London: Hogarth Press.

Broida, J., Tingley, L., Kimball, R., \& Miele, J. (1993). Personality differences between pro-and antivivisectionists. Society and Animals, 1, 129-144. http://dx.doi.org/10.1163/156853093X00037

Cassels, T. G., Chan, S., Chung, W., \& Birch, S. A. J. (2010). The role of culture in affective empathy: Cultural and bicultural differences. Journal of Cognition and Culture, 10, 309-326. http://dx.doi.org/10.1163/156853710X531203

Castro, R., Gaspar, A., \& Vicente, L. (2010). The Evolving empathy: hardwired bases of human and nonhuman primate empathy. Psicologia, XXIV(2), 131-152.

Christov-Moore, L., Simpson, E. A., Coude, G., Grigaityte, K., Iacoboni, M., \& Ferrari, P. F. (2014). Empathy: Gender effects in brain and behavior. Neuroscience and Biobehavioral Reviews, 46, 604-627. http://dx.doi.org/10.1016/j.neubiorev.2014.09.001

Clary, E. G., \& Snyder, M. (1999). The motivations to volunteer. Theoretical and Practical Considerations. Current Directions in Psychological Science, 8(5), 156-159. http://dx.doi.org/10.1111/14678721.00037

Daly, B., \& Morton, L. L. (2006). An investigation of human-animal interactions and empathy as related to pet preference, ownership, attachment, and attitudes in children. Anthrozoös, 19(2), 113-128. http://dx.doi.org/10.2752/089279309X12538695316383

Davis, M. H. (1980). A multidimensional approach to individual differences in empathy. JSAS Catalog of Selected Documents in Psychology, 10, 85.

Davis, M. H. (1994). Empathy: A social psychological approach. Madison, WI: Brown and Benchmark.

de Waal, F. B. M. (2008). Putting the altruism back into altruism: the evolution of empathy. Annual Review of Psychology, 59, 279-300. http://dx.doi.org/10.1146/annurev.psych.59.103006.093625 
Decety, J. \& Svetlova, M. (2012). Putting together phylogenetic and ontogenetic perspectives on empathy. Developmental cognitive neuroscience, 2(1), 1-24. http://dx.doi.org/10.1016/j.dcn.2011.05.003

Eddy, T. J., Gallup, G. G., \& Povinelli, D. J. (1993). Attribution of cognitive states to animals: Anthropomorphism in comparative perspective. Journal of Social Issues, 49(1), 87-101. http://dx.doi.org/10.1111/j.1540-4560.1993.tb00910.x

Eisenberg, N. (2000). Empathy and sympathy. In M. Lewis \& J. M. Haviland-Jones (2nd ed), Handbook of Emotion (pp. 677-91). New York: Guilford.

Eisenberg, N., Fabes, R. A., Miller, P. A., Fultz, J., Mathy, R. M., Shell, R., \& Reno, R. R. (1989). Relation of sympathy and personal distress to prosocial behavior: A multimethod study. Journal of Personality and Social Psychology, 57(1), 55-66. http://dx.doi.org/10.1037/0022-3514.61.3.459

Eisenberg, N., Miller, P. A., Shell, R., McNalley, S., \& Shae, C. (1991). Prosocial development in adolescence: A longitudinal study. Developmental Psychology, 27(5), 849-857. http://dx.doi.org/10.1037/00121649.27.5.849

Ellingsen, K., Zanella, A. J., Bjerkås, E., \& Indrebø, A. (2010). The relationship between empathy, perception of pain and attitudes toward pets among Norwegian dog owners. Anthrozoös, 23(3), 231-243. http://dx.doi.org/10.2752/175303710X12750451258931

Emauz, A., Gaspar, A., Esteves, F., \& Carvalhosa, S. F. (2016). Adaptação da Escala de Empatia pelos Animais (EEA) para a população portuguesa. Análise Psicológica, vol. XXXIV, 189-201. http://dx.doi.org/10.14417/ap.1049

Filippi, M., Riccitelli, G., Falini, A., Di Salle, F., Vuilleumier, P., Comi, G., \& Rocca, M. A. (2010). The brain functional networks associated to human and animal suffering differ among omnivores, vegetarians and vegans. PloS One, 5(5), e10847. http://dx.doi.org/10.1371/journal.pone.0010847

Fox, N., \& Ward, K. (2008). Health, ethics and environment: A qualitative study of vegetarian motivations. Appetite, 50(2-3), 422-429. http://dx.doi.org/10.1016/j.appet.2007.09.007

Furnham, A., McManus, C., \& Scott, D. (2003). Personality, empathy and attitudes to animal welfare. Anthrozoös, 16(2), 135-146. http://dx.doi.org/10.2752/089279303786992260

Gaspar, A. (2014). Neurobiologia e psicologia da empatia. Pontos de partida para a investigação e intervenção da promoção da empatia. In P. Henenberg and A. C. Caldas (Eds), Cérebro: $O$ que a ciência nos diz. Povos e Culturas 18 (pp. 159-174). Lisboa: Universidade Católica Editora.

Harris, P. L. (2000). Children's understanding of emotion ( $3^{\text {rd }}$ Ed.). In M. L. Lewis, J.M: Haviland-Jones and L. Barret (Eds.), Handbook of Emotions (pp. 320- 363). New York: The Guilford Press.

Hawkins, R. D., Williams, J. M., \& Scottish Society for the Prevention of Cruelty to Animals (Scottish SPCA) (2017). Childhood attachment to pets: Associations between pet attachment, attitudes to animals, compassion, and humane behaviour. International Journal of Environmental Research and Public Health, 14(5), 490. http://dx.doi.org/10.3390/ijerph14050490

Herzog, H. (2007). Gender differences in human-animal interactions: A review. Anthrozoös, 20(1), 7-21. http://dx.doi.org/10.2752/089279307780216687

Herzog, H., Betchart, N., \& Pittman, R. (1991). Gender, sex role identity and attitudes toward animals. Anthrozoös, 4(3), 184-192. http://dx.doi.org/10.2752/089279391787057170

Hills, A. (1995). Empathy and belief in the mental experience of animals. Anthrozoös, 8(3), 132-142. http://dx.doi.org/10.2752/089279395787156347

Hoffman, M. L. (1975). Altruistic behavior and the parent- child relationship. Journal of Personality and Social Psychology, 31, 937-943. http://dx.doi.org/http://dx.doi.org/10.1037/h0076825

Hrdy, S. B. (2011) Mothers and Others: the evolutionary origins of mutual understanding. Cambridge, MA: Harvard University Press.

Kavanagh, P. S., Signal, T. D., \& Taylor, N. (2013). The dark triad and animal cruelty: Dark personalities, dark attitudes, and dark behaviors. Personality and Individual Differences, 55(6), 666-670. http://dx.doi.org/10.1016/j.paid.2013.05.019

Kean, H. (1998). Animal rights: Political and social change in Britain since 1800. London: Reaktion Books.

Knight, S., \& Barnett, L. (2008). Justifying attitudes toward animal use: A qualitative study of people's views and beliefs. Anthrozoös, 21(1), 31-42. http://dx.doi.org/10.2752/089279308X274047

Knight, S., Nunkoosing, K., Vrij, A., \& Cherryman, J. (2003). Using grounded theory to examine people's attitudes toward how animals are used. Society \& Animals, 11(4), 307-327. http://dx.doi.org/10.1163/156853003322796064

Komorosky, D., \& O'Neal, K. K. (2015). The development of empathy and prosocial behavior through humane education, restorative justice, and animal-assisted programs. Contemporary Justice Review: Issues in Criminal, Social, and Restorative Justice, 18(4), 395-406. http://dx.doi.org/10.1080/10282580.2015.1093684 
Limpo, T., Alves, R. A., \& Castro, S. L. (2010). Medir a empatia: Adaptação portuguesa do Índice de Reactividade Interpessoal. Laboratório de Psicologia, 8(2), 171-184. Retirado de http://publicacoes.ispa.pt/index.php/lp/article/viewFile/640/628

Lindzey, A. \& Clarke, P.B. (2004). Animal rights: A historical anthology. NY: Columbia University Press.

MacFarland, D. (1987). The Oxford companion to animal behaviour. Oxford, UK: Oxford University Press.

Mathews, S., \& Herzog, H. (1997). Personality and attitudes towards the treatment of animals. Society \& Animals, 5(2), 57-63. http://dx.doi.org/10.1163/156853097X00060

McPhedran, S. (2009). A review of the evidence for associations between empathy, violence, and animal cruelty. Aggression and Violent Behavior, 14(1), 1-4. http://dx.doi.org/10.1016/j.avb.2008.07.005

Mehrabian, A., \& Epstein, N. (1972). A measure of emotional empathy. Journal of Personality, 40(4), 525543. http://dx.doi.org/10.1086/521907

Melson, G. (2003). Child development and the human-companion animal bond. American Behavioral Scientist, 47, 31-39. http://dx.doi.org/10.1177/0002764203255210

Mestre, M. V., Samper, P., Frías, M. D., \& Tur, A. M. (2009). Are women more empathetic than men? A longitudinal study in adolescence. The Spanish Journal of Psychology, 12(1), 76-83. http://dx.doi.org/10.1017/S1138741600001499

Nagasawa, M., Kikusui, T., Onaka, T., \& Ohta, M. (2009). Dog's gaze at its owner increases owner's urinary oxytocin during social interaction. Hormones and Behavior, 55(3), 434-441. http://dx.doi.org/10.1016/j.yhbeh.2008.12.002

Nielsen (2007). Estudo para o Centro Vegetariano para determinar o número de vegetarianos em Portugal. Retirado de http://www.centrovegetariano.org/Article-451Portugal\%253A\%2B30\%2B000\%2BVegetarianos.html

O’Brien, E., Konrath, S. H., Grühn, D., \& Hagen, A. L. (2013). Empathic concern and perspective taking: Linear and quadratic effects of age across the adult life span. The Journals of Gerontology Series B: Psychological Sciences and Social Sciences, 68(2): 168-175. http://dx.doi.org/10.1093/geronb/gbs055

Paul, E. (2000). Empathy with animals and with humans: Are they linked? Anthrozoös, 13(4), 194-202. http://dx.doi.org/10.2752/089279300786999699

Paul, E. (2005). Love of pets and love of people. In A. L. Podberscek, E. P. Paul \& J. A. Serpell (Eds.), Companion animals and us. Exploring the relationships between people and pets (pp. 168-186). New York, NY: Cambridge University Press.

Paul, E. S., \& Serpell, J. A. (1993). Childhood pet keeping and humane attitudes in young adulthood. Animal Welfare, 2, 321-337.

Pręgowski, M. P. (2016). Companion animals in everyday life. (M. P. Pręgowski, Ed.) New York, NY: Palgrave Macmillan. http://dx.doi.org/10.1057/978-1-137-59572-0

Preston, S. D., \& de Waal, F. B. M. (2002). Empathy: Its ultimate and proximate bases. The Behavioral and Brain Sciences, 25(1), 1-20; discussion 20-71. Retirado de http://www.ncbi.nlm.nih.gov/pubmed/12625087

Regan, T. (1983). The case for animal rights. Berkeley: University of California Press.

Rocha, S., Gaspar, A., \& Esteves, F. (2016) Developing children's ability to recognize animal emotions what does it take? A study at the Zoo. Human Animal Interaction Bulletin, 4(2), 59-79.

Rodrigues, S. M., Slaslow, L. R., Garcia, N., John, O. P., \& Keltner, D. (2009). Oxytocin receptor genetic variation relates to empathy and stress reactivity in humans. Proceedings of the National Academy of Science of the United States of America, 106(50), 21437-21441. http://dx.doi.org/10.1073/pnas.0909579106.

Rothgerber, H., \& Mican, F. (2014). Childhood pet ownership, attachment to pets, and subsequent meat avoidance. The mediating role of empathy toward animals. Appetite, 79, 11-17. http://dx.doi.org/10.1016/j.appet.2014.03.032

Schieman, S., \& Gundy, K. V. (2000). The personal and social links between age and self-reported empathy. Social Psychology Quarterly, 63(2), 152. http://dx.doi.org/10.2307/2695889

Schore, A. N. (2001). Effects of a secure attachment relationship on right brain development, affect regulation, and infant mental health. Infant Mental Health Journal, 22(1-2), 7-66. http://dx.doi.org/10.1002/1097-0355(200101/04)22:1<7::AID-IMHJ2>3.0.CO;2-N

Signal, T., \& Taylor, N. (2006). Attitudes to Animals: Demographics Within a Community Sample. Society \& Animals, 14(2), 147-157. http://dx.doi.org/10.1163/156853006776778743

Singer, P. (2015). The most good you can do: How effective altruism is changing ideas about living ethically. New Haven: Yale University Press.

Smith, A. (2006). Cognitive empathy and emotional empathy in human behavior and evolution. The Psychological Record, 56, 3-21. https://doi.org/10.1007/BF03395534 
Szűcs, E., Geers, R., Jezierski, T., Sossidou, E. N., \& Broom, D. M. (2012). Animal welfare in different human cultures, traditions and religious faiths. Asian-Australasian Journal of Animal Sciences, 25(11), 1499-1506. http://doi.org/10.5713/ajas.2012.r.02

Taylor, N., \& Signal, O. (2005). Empathy and attitudes to animals. Anthrozoös, 18, 18-27. . http://doi.org/10.2752/089279305785594342

Thompson, K. L., \& Gullone, E. (2008). Prosocial and antisocial behaviors in adolescents: An investigation into associations with attachment and empathy. Anthrozoös, 21(2), 123-137. http://dx.doi.org/10.2752/175303708X305774

Toussaint, L., \& Webber, J. R. (2005). Gender differences in the relationship between empathy and forgiveness. The Journal of Social Psychology, 145(6), 673-685. http://dx.doi.org/10.3200/SOCP.145.6.673-686

Trommsdorff, G., Friedlmeier, W., \& Mayer, B. (2007). Sympathy, distress and prosocial behaviour of preschool children in four cultures. International Journal of Behavioural Development, 31, 284-293. http://dx.doi.org/10.1177/0165025407076441

Vegetarian Times (2016, Outubro 11). Vegetarianism in America. Retirado de http://www.vegetariantimes.com/article/vegetarianism-in-america

Wagstaff, G. (1991). Attitudes toward animals and human beings. Journal of Social Psychology, 131, 573575. http://dx.doi.org/10.1080/00224545.1991.9713887

Wells, D. L., \& Hepper, P. J. (1997). Pet ownership and adults' views on the use of animals. Society \& Animals, 5(1), 45-63. https://doi.org/10.1163/156853097X00213

Westbury, H. R., \& Neumann, D. L. (2008). Empathy-related responses to moving film stimuli depicting human and non-human animal targets in negative circumstances. Biological Psychology, 78(1), 6674. http://dx.doi.org/10.1016/j.biopsycho.2007.12.009

Zahn-Waxler, C., \& Radke-Yarrow, M. (1990). The Origins of empathic concern. Motivation and Emotion, 14(2), 107-130. http://dx.doi.org/10.1007/BF00991639

Historial do artigo

Recebido 14/08/2016

Aceite $\quad 13 / 10 / 2017$

Publicado 05/2018 